\title{
Single Term Off-Line Coins
}

\author{
Niels Ferguson \\ CWI, P.O. Box 94079, 1090 GB Amsterdam, Netherlands. \\ e-mail: nielsecwi.nI
}

\begin{abstract}
We present a new construction for off-line electronic coins that is both far more efficient and much simpler than previous systems. Instead of using many terms, each for a single bit of the challenge, our system uses a single term for a large number of possible challenges. The withdrawal protocol does not use a cutand-choose methodology as with earlier systems, but uses a direct construction.
\end{abstract}

\section{Introduction}

The main requirements for an electronic cash system are the following:

- Security. Every party in the electronic cash system should be protected from a collusion of all other parties (multi-party security).

- Off-line. There should be no need for communication with a central authority during payment.

- Fake Privacy. The bank and all the shops should together not be able to derive any knowledge from their protocol transcripts about where a user spends her money.

- Privacy (untraceable). The bank should not be able to determine whether two payments were made by the same payer, even if all shops cooperate.

The privacy requirement is obviously stronger then the fake privacy one. Under real-world circumstances, a payer is identified during some of the payments by means outside the electronic cash system. If two payments can be recognised as originating from the same payer, then knowing the identity of the payer during a single payment anywhere allows the tracing of all other payments made by that payer. Therefore, fake privacy provides no privacy at all in practice.

Electronic cash was first introduced by Chaum, Fiat and Noar [CFN90]. Their system (later improved in $\left[\mathrm{CdBvH}^{+} 90\right]$ ) meets all of the requirements, but is quite complex. The authors give a construction for electronic checks that allow a variable amount of money to be payed with a single signature. These checks can be thought of as a bunch of fixed-value coins sharing common overhead.

In [vA90] Hans van Antwerpen described a different scheme which is more efficient than [CFN90] and [CdBvH ${ }^{+90}$, but has the same basic properties.

Okamoto and Ohta introduced the idea of divisibility of electronic cash [0092]. This allows a piece of money to be divided into smaller pieces each 
of which can be spent separately. Their construction satisfies all of the above requirements except the privacy, and the fake privacy of the user is only computationally protected.

The most difficult fraud to counter in electronic cash systems is doublespending. A user can always spend a single coin twice in two different shops. This fraud cannot be detected at the time of spending as payments are off-line. The solution that all electronic cash systems use is to detect the double-spending after the fact. At each payment the user is required to release information in response to a challenge from the shop. One such release provides no clue to the user's identity, but two such releases are sufficient to identify the user uniquely.

In all earlier schemes, the identification of double-spenders requires multiple terms in each coin. Each term is used to answer one bit of challenge from the shop during payment. If both possible answers for any term is ever given, the user's identity is revealed. To achieve an acceptable probability of detection, a large number of terms is required. These schemes are therefore inefficient if a piece of money has a fixed value, so all of them provide some way to pay a variable amount of money with a single 'check'. The resulting refunds for partially spent checks pose further security and privacy problems [Hir93] as well as user-interfacing problems. Another major disadvantage of check systems is that they are very complex (the full but concise mathematical description of the protocols in [vA90] requires 40 pages), which makes them extremely difficult to understand, verify, implement or debug.

Recently, in independent work, Franklin and Yung gave a construction for a provably secure coin scheme in a slightly different setting where a trusted centre is available to produce 'blank' coins [FY93]. An alternative they discuss does not require the trusted centre, but is not provably secure. Like our construction, they use a secret sharing line instead of multiple challenge terms.

Up to this point all electronic cash schemes have used a cut-and-choose protocol for constructing the money. These protocols are by their very nature inefficient. To get a low enough probability of cheating, the cut-and-choose must consist of many terms, half of which are thrown away.

Our new system (described earlier in [Fer93]) is a coin scheme where each piece of money has a fixed value. Each coin consists of 3 numbers plus 2 RSA signatures and can be stored in about 250 bytes. (This assumes that we multiply the signatures of 4 different coins together to save storage space; recovering the original signatures is easy [Cha90].) During payment (of possibly several coins) the user has to perform about $\mathbf{3 0}$ modular multiplications plus two multiplications per coin being payed. The withdrawal protocol constructs the coins directly without resorting to cut-and-choose methods.

In [Bra93, Bra94] Stefan Brands recently showed a different construction for an efficient electronic coin scheme based on discrete logarithms. His construction does not use a cut-and-choose protocol or multiple challenge terms either.

I would like to thank David Chaum for all his support, and for helping to clean up and improve the withdrawal protocol. Stefan Brands and Ronald Cramer provided many helpful comments. 


\section{Efficient payments}

Instead of using many challenge terms for double-spending detection we will use a polynomial secret sharing scheme [Sha79]. The user (Alice) gives a share of her identity to the shop at each payment in response to a random challenge. If Alice spends the same coin twice, she has to give two different shares which will allow the bank to recover her identity eventually. This solution gets rid of the large number of challenge terms in all previous systems.

The central idea (developed in cooperation with Hans van Antwerpen) is to represent a coin as 3 numbers, $C:=f_{c}(c), A:=f_{a}(a)$, and $B:=f_{b}(b)$ where the $f$ functions are suitable oneway functions. Alice also gets two RSA-signatures from the bank: $\left(C^{k} A\right)^{1 / v}$ and $\left(C^{U} B\right)^{1 / v}$, where $v$ is a prime, $U$ is Alice's identity and $k$ is a random number.

During payment, Alice sends the numbers $c, a$, and $b$ to the shop. The shop replies with a randomly chosen (non-zero) challenge $x$. Finally Alice sends $r:=k x+U(\bmod v)$ (which is the share of her identity) and the signature $\left(C^{r} A^{x} B\right)^{1 / v}$ which she can easily compute from the two signatures she has. The shop, in turn, can verify the consistency of these two responses. When spending several coins at the same time, the same challenge is used for all the coins and Alice sends the product of all the signatures to the shop. (Coins of different values use a different $v$.) This reduces the computations done by Alice to two multiplications per coin plus one exponentiation to the power $x$. For a 20-bit challenge the exponentiation requires about 30 multiplications.

The payment protocol can obviously be converted to a one-move protocol by choosing $x$ as a hash value on the coin(s) and the shops identity. This requires a larger challenge but eliminates the interaction between the payer and payee.

At the end of the day, the shop sends $c, a, b$, the challenge and the response to the bank. The bank can verify the correctness of the coin and credit the shop with the corresponding amount. If Alice spends the same coin twice, she must reveal two different points on the line $x \mapsto k x+U$ which immediately allows the bank to determine her identity $U$.

Given this idea for the payment protocol, the problem of withdrawing the coin from the bank remains. Alice must get the signatures on $C^{k} A$ and $C^{U} B$ while the bank learns nothing about $c, a, b$ or $k$.

\section{Randomized blind signatures}

For our construction of an efficient withdrawal protocol we need a signature scheme with the following properties:

- Alice receives an RSA-signature on a number of a special form, which she cannot create herself.

- The bank is sure that the number it signs was randomly chosen.

- The bank receives no information regarding which signature Alice gets. 
We call such a signature a randomized blind signature. The scheme that we use here is due to David Chaum [Cha92].

The protocol to get a randomized blind signature is shown in Fig. 1. All computations are done in an RSA system [RSA78] where the bank knows the factorization of the modulus $n$. The public exponent of the RSA system is $v$, a reasonably large prime (say 128 bits). Alice starts by choosing a random $a_{1}$, and two blinding factors $\sigma$ and $\gamma$. She computes $\gamma^{v} a_{1} g^{\sigma}$ where $g$ is a (publicly known) element of large order in $\mathbb{Z}_{n}^{*}$ and sends the result to the bank. The bank chooses its own contribution $a_{2}$ and sends this back to Alice. Alice replies with $f\left(a_{1} a_{2}\right)-\sigma$ where $f(\cdot)$ is a suitable oneway function mapping $\mathbb{Z}_{n}^{*}$ into $\mathbb{Z}_{v}$. The bank multiplies $\gamma^{v} a_{1} g^{\sigma}$ by $a_{2}$ and $g^{f\left(a_{1} a_{3}\right)-\sigma}$ to get $\gamma^{v} a_{1} a_{2} g^{f\left(a_{1} a_{2}\right)}$, computes the $v^{\prime}$ th root of this number and sends it to Alice. Alice divides out the blinding factor $\gamma$ to get the pair $\left(a,\left(a g^{f(a)}\right)^{1 / v}\right)$. The number $a$ is called the base number of the signature.

$$
\begin{aligned}
& \text { Alice } \\
& \text { Bank } \\
& a_{1}, \gamma \in \mathcal{R} \mathbb{Z}_{n}^{*} \\
& \sigma \in_{\mathbb{R}} \mathbb{Z}_{\boldsymbol{v}} \\
& \gamma^{v} a_{1} g^{\sigma} \\
& a_{2} \in \mathbb{R}_{\mathcal{R}} \mathbb{Z}_{n}^{*} \\
& e \leftarrow f\left(a_{1} \cdot a_{2}\right)-\sigma \stackrel{\frac{a_{2}}{e^{\longrightarrow}}}{\bar{A} \leftarrow \gamma^{v} a_{1} g^{\sigma} \cdot a_{2} \cdot g^{\circ}} \\
& (\bar{A})^{1 / v} \\
& a \leftarrow a_{1} a_{2} \\
& S \leftarrow(\bar{A})^{1 / v} / \gamma \\
& S^{\nu} \stackrel{?}{=} a g^{f(\alpha)}
\end{aligned}
$$

Fig. 1. Randomized blind signature scheme

Note: To make the blinding perfect, all computations involving exponents are done modulo $v$. For example, $e$ is computed as $\left(f\left(a_{1} a_{2}\right)-\sigma\right) \bmod v$. Alice can correct for the possible additional factor of $g^{v}$ by multiplying the final signature by $g^{(f(a)-\sigma) \text { div } v}$. In the rest of this paper we will assume implicitly that all computations involving exponents are done modulo $v$ and that the necessary corrections are applied to the resulting signatures.

Assumption 1. It is computationally infeasible to forge a signature pair of the form $\left(a,\left(a g^{f(a)}\right)^{1 / v}\right)$. 
Reasoning. This assumption is a special case of the RSA signature assumption. Suppose Alice tries to forge a signature pair $(a, A)$. If we define $t:=f(a)$, then she must have solved the equation $t=f\left(A^{v} g^{-t}\right)$. Two ways to solve this equation spring to mind. The first one is to choose $A$ and then try different values of $t$ until you get lucky (probability of success is $1 / v$ ). The second one is to choose $A^{v} g^{-t}$, compute $t$, and then try to compute $A$. This requires the computation of a $v^{\prime}$ th root. Neither of these methods seems feasible.

Even if Alice has a large number of valid signature pairs, it is still difficult for her to find new ones. A result of Evertse and van Heyst [EvH92] implies (loosely stated) that the only new RSA signatures that can be computed from old ones are multiplicative combinations of the old signatures. If you multiply two signatures of the form $a g^{f(a)}$, you do not get another valid signature, unless $f(a b)=f(a)+f(b)$. One of the requirements for $f$ is that it is infeasible to find relations of this kind.

Proposition 2. The bank gets no information regarding $\left(a,\left(a g^{f(a)}\right)^{1 / v}\right)$ from the protocol in Fig. 1.

Proof. We define the view of the bank as all the communication to and from the bank, plus all the random choices that the bank made. Given the banks view of a run of the protocol, we will show that for every legal pair $(a, A)$ there is exactly one set of random choices that Alice could have made which would result in her receiving that signature in that protocol run. This makes all possible signature pairs equally likely, given the knowledge of the bank.

Given $a$ (from the pair) and $a_{2}$ (from the view), $a_{1}$ must have been chosen as $a / a_{2}$. Alice's choice of $\sigma$ is given by subtracting the value in the third transmission $(f(a)-\sigma)$ from $f(a)$ (computed from $a)$. Finally, Alice's choice of $\gamma$ is uniquely determined by the first transmission.

If Alice had indeed chosen $a_{1}, \sigma$ and $\gamma$ in this way, then she would have gotten $(a, A)$ as a signature pair. So, from the banks point of view, all signature pairs are equally likely.

\section{1 'Abuses' by Alice}

There are several ways in which Alice can deviate from this protocol which we will investigate briefly. For this we rewrite the protocol as shown in Fig. 2. We assume that Alice is choosing the numbers $A-E$ in some clever way. For practical reasons we have to restrict Alice's behaviour a bit. In choosing the numbers, she can use any construction, but we assume that the only computations that she does with the final signature are an exponentiation and a multiplication. (Other operations don't seem to make sense on an RSA signature.) Furthermore, Alice should end up with a $v^{\prime}$ th root on a number of the form $K g^{f(K)}$ for some $K$ that Alice can compute. Any other results are not of interest in the coin system.

First of all, observe that $B$ only occurs as $C+B$ in the result. As $C$ is chosen later then $B$, we can assume $B=0$ without loss of generality. 
Alice

Bank

$A \in \mathbb{Z}_{n}^{*}$

$B \in \mathbb{Z}_{v}$

$\begin{array}{ll}C \in \mathbb{Z}_{v} & \frac{A g^{B}}{a_{2}} a_{2} \in \mathbb{Z}_{n}^{*} \\ & \frac{C}{\left(A a_{2} g^{B+C}\right)^{1 / v}} \\ D \in Z_{n}^{*} & \\ E \in Z_{v} & \\ R \leftarrow D\left(A a_{2} g^{B+C}\right)^{E / v}\end{array}$

Fig. 2. Possible behaviours of Alice

To get a useful signature, Alice must have $R=\left(K g^{f(K)}\right)^{1 / v}$, which is equivalent to $D^{v} A^{E} a_{2}^{E} g^{E C}=K g^{f(K)}$. We can assumed that Alice doesn't use a factor $g^{x}$ in $A$ for some $x$. (Alice can get the same effect by adding $x$ to $C$.) The exponents on $g$ are modulo $v$, so $D$ cannot contribute to them. As Alice doesn't know $g^{1 / v}$, she cannot put any extra factors in herself. To get something useful she must therefore solve the following two simultaneous equations.

$$
\begin{aligned}
t+E C & =f(K) \quad(\bmod v) \\
D^{v} A^{E} a_{2}^{E} & =K g^{t}
\end{aligned}
$$

for some $t \in \mathbb{Z}_{v}$. There seems only one way to do this, and that is to fix $K$ by choosing $D, A, E$ and $t$, and then choosing $C$ to fit the first equation. (Any other way would involve inverting the oneway function, or computing a root.) But this means that $D$ and $E$ must have been fixed before sending $C$ to the bank.

We conclude that Alice can 'abuse' this protocol by sending a slightly different reply in the third message. She can choose any $D$ and $E$ such that she gets a valid signature (on a number of the form $x g^{f(x)}$ ) after raising it to the $E^{\prime}$ th power and multiplying it by $D$. The factor $D$ doesn't help Alice much in cheating. Alice can modify the base number after the bank has revealed $a_{2}$, but she can only multiply the base number by $D^{v}$. Because Alice cannot compute roots, any $v^{\prime}$ th power is essentially random to her, thus she cannot control the way in which she changes the base number. Alice could at most use the $D$ factor to shift the uniform distribution of the base number slightly, but in the large set of possible base numbers this is hardly significant. The same basically holds for the $E$ power.

Unfortunately, we cannot prove that there is no other way for Alice to cheat. The attacks allowed in Fig. 2 are only the most obvious ones. There might for 
example be an attack in which Alice computes the cosine of the last reply to get something useful, but this seems somewhat unlikely to give any useful result. At present, the state of the art in cryptography does not allow us in general to prove the security of such a protocol.

When $E \neq 1$ it is essential for Alice to be able to compute $a_{2}^{E}$ after receiving $a_{2}$. For our coin withdrawal protocol we also need a restricted version (see Fig. 3) which does not allow Alice to choose $E \neq 1$. Instead of $a_{2}$, the bank sends $h^{a_{2}} \bmod p$ where $p$ is a prime congruent to 1 modulo $n$, and $h$ is a publicly known element of order $n$ in $\mathbb{F _ { p }}$. The exponents of $h$ are thus reduced modulo $n$ so the numbers in the exponent behave in exactly the same way as in the RSA system. The final form of the signature will not be $\left(a g^{f(a)}\right)^{1 / v}$ but rather $\left(a g^{f\left(h^{a}\right)}\right)^{1 / v}$. Because Alice cannot compute $h^{a_{2}^{E}}$ given only $h^{a_{2}}$ she can no longer choose $E$.

$$
\begin{aligned}
& \text { Alice Bank } \\
& a_{1}, \gamma \in \mathcal{R} \mathbb{Z}_{n}^{*} \\
& \sigma \in \in_{\mathbb{R}} \mathbb{Z}_{v} \\
& \stackrel{\gamma^{v} a_{1} g^{\sigma}}{\longrightarrow} \quad a_{2} \in \mathcal{R} \mathbb{Z} \\
& e \leftarrow f\left(h^{a_{1} a_{2}}\right)-\sigma \stackrel{\frac{h^{a_{2}}}{e^{\longrightarrow}} \bar{A} \leftarrow \gamma^{v} a_{1} g^{\sigma} \cdot a_{2} \cdot g^{e}}{\longrightarrow} \\
& \stackrel{a_{2},(\bar{A})^{1 / v}}{\longleftarrow} \\
& a \leftarrow a_{1} a_{2} \\
& S \leftarrow(\bar{A})^{1 / v} / \gamma \\
& S^{v} \stackrel{?}{=} a g^{f\left(h^{a}\right)}
\end{aligned}
$$

Fig. 3. Randomized blind signature scheme without exponential attack

\section{Coin withdrawal protocol}

For our system we need 3 numbers, $C, A$, and $B$. These will be of the form

$$
\begin{aligned}
& C=c g_{c}^{f\left(h_{c}^{c}\right)} \\
& A=a g_{a}^{f(a)} \\
& B=b g_{b}^{f\left(h_{b}^{b}\right)}
\end{aligned}
$$

where the numbers $g_{c}, g_{a}$ and $g_{b}$ are publicly known and of large order in the group $Z_{n}^{*}$. The numbers $h_{c}$ and $h_{b}$ are elements of order $n$ from $\mathbb{F}_{p}$ where $p-1$ 
is a multiple of $n$. The use of three different $g$ values ensures that the numbers are distinct and do not mingle when multiplied together in a signature.

The coin withdrawal protocol (see Fig. 4) consists of three parallel runs of the randomized blind signature scheme. Two of the runs are the restricted version, and one is the unrestricted version. The $E$ exponent is used to allow Alice to randomise the $k$ parameter of the secret sharing line herself while the bank can ensure that the other parameter is Alice's identity $U$.

$$
\begin{aligned}
& \text { Alice } \\
& \text { Bank } \\
& c_{1}, a_{1}, b_{1} \in \mathcal{R} \mathbb{Z}_{n}^{*} \\
& \sigma, \tau, \phi \in \mathbb{R} \mathbb{Z}_{\boldsymbol{v}} \\
& \gamma, \alpha, \beta \in \in_{\mathcal{R}} \mathbb{Z}_{n}^{*} \\
& \stackrel{\gamma^{v} c_{1} g_{c}^{\sigma}, \alpha^{v} a_{1} g_{a}^{\tau}, \beta^{v} b_{1} g_{b}^{\phi}}{h_{c}^{c_{2}}, a_{2}, h_{b}^{b_{2}}} c_{c_{2}, a_{2}, b_{2} \in \mathcal{R}} \mathbb{Z}_{n}^{*} \\
& \stackrel{\gamma^{v} c_{1} g_{c}^{\sigma}, \alpha^{v} a_{1} g_{a}^{\tau}, \beta^{v} b_{1} g_{b}^{\phi}}{h_{c}^{c_{2}}, a_{2}, h_{b}^{b_{2}}} c_{c_{2}, a_{2}, b_{2} \in \mathcal{R}} \mathbb{Z}_{n}^{*} \\
& k_{1} \in \in_{R} \mathbb{Z}_{v}^{*} \\
& e_{c} \leftarrow f\left(h_{c}^{c_{1} c_{2}}\right)-\sigma \\
& e_{b} \leftarrow f\left(h_{b}^{b_{1} b_{2}}\right)-\phi \\
& a \leftarrow\left(a_{1} a_{2} \cdot f_{2}\left(e_{c}, e_{b}\right)\right)^{k_{1}} \\
& e_{a} \leftarrow \frac{1}{k_{1}} f(a)-\tau \\
& \begin{array}{r}
e_{c, e_{a}, e_{b}} \\
\bar{C} \leftarrow \gamma^{v} c_{1} g_{c}^{\sigma} \cdot c_{2} \cdot g_{c}^{e_{c}} \\
\bar{A} \leftarrow \alpha^{v} a_{1} g_{a}^{\top} \cdot a_{2} \cdot f_{2}\left(e_{c}, e_{b}\right) \cdot g_{a}^{e_{a}} \\
\bar{B} \leftarrow \beta^{v} b_{1} g_{b}^{\phi} \cdot b_{2} \cdot g_{b}^{e_{b}} \\
k_{2} \in_{\mathcal{R}} \mathbb{Z}_{v}^{*}
\end{array} \\
& c \leftarrow c_{1} c_{2} \\
& b \leftarrow b_{1} b_{2} \\
& k \leftarrow k_{1} k_{2} \bmod v \\
& C \leftarrow c g_{\mathrm{c}}^{f\left(h_{c}^{c}\right)} \\
& A \leftarrow a g_{a}^{f(a)} \\
& B \leftarrow b g_{b}^{f\left(h_{b}^{b}\right)} \\
& S_{\alpha} \leftarrow\left(\left(\bar{C}^{k_{2}} \cdot \bar{A}\right)^{1 / v} / \gamma^{k_{2}} \alpha\right)^{k_{1}} \\
& S_{b} \leftarrow\left(\bar{C}^{U} \cdot \bar{B}\right)^{1 / v} / \gamma^{U} \beta \\
& S_{a}^{v} \stackrel{?}{=} C^{k} A \\
& S_{b}^{u} \stackrel{?}{=} C^{U} B \text {. }
\end{aligned}
$$

Fig. 4. Coin withdrawal protocol 
There are 2 additions to this simple parallel-run view. One is that there is an extra one-way function that makes $a$ depend on $e_{c}$ and $e_{b}$. This prevents Alice form choosing $e_{c}$ and $e_{b}$ as a function of $a$. Were she able to do this, she could cancel some of the terms and get a signature on just $C$ and $B$. Although this is not a threat as such against the payment scheme, it is undesirable that Alice has so much freedom.

The second modification is that the bank puts a random power on $C$ in the first signature. Alice is going to end up with two signatures: $\left(C^{k} A\right)^{1 / v}$ and $\left(C^{U} B\right)^{1 / v}$. Here, $U$ is the identity and $k$ is a random number unknown to the bank. However, to prevent Alice from combining an old coin with the one currently being withdrawn the bank must ensure that $k$ is indeed random. Therefore, the bank puts a random power on the $C$ in the first signature, forcing $k$ to be random. The protocol consists of the following steps:

1. Alice starts by choosing the random numbers $c_{1}, a_{1}, b_{1}, \sigma, \tau, \phi, \alpha, \beta$, and $\gamma$. The first three are Alice's contributions to the base numbers. The second triple are the exponential blinding factors, and the third triple are the multiplicative blinding factors. Alice computes $\gamma^{v} c_{1} g_{c}^{\sigma}, \alpha^{v} a_{1} g_{a}^{\tau}$ and $\beta^{v} b_{1} g_{b}^{\phi}$, and sends these numbers to the bank.

2. The bank then chooses its three contributions to the base numbers $c_{2}, a_{2}$, $b_{2}$. It sends $h_{c}^{c_{2}}, a_{2}$, and $h_{b}^{b_{2}}$ to Alice.

3. Alice chooses a random number $k_{1}$, and computes the exponents $e_{c}$ and $e_{b}$ as $f\left(h_{c}^{c_{1} c_{2}}\right)-\sigma$ and $f\left(h_{b}^{b_{1} b_{2}}\right)-\phi$. She computes $a$ as $\left(a_{1} a_{2} f_{2}\left(e_{c}, e_{b}\right)\right)^{k_{1}}$ where $f_{2}(\cdot)$ is a suitable oneway function. The exponent $e_{\alpha}$ is computed somewhat differently to get the right exponent after raising the signature to the $k_{1}$ 'th power. After computing $e_{a}$ as $\left(1 / k_{1}\right) f(a)-\tau$, Alice send all three exponents to the bank. Note: The subtractions and multiplication by $1 / k_{1}$ are done modulo $v$. Any modulo reduction here has to be corrected in the final signature, by multiplying the signature by a suitable powers of $g_{c}, g_{a}$ and $g_{b}$. These corrections are not shown.

4. The bank now computes the blinded versions of $C, A$ and $B . \bar{C}$ is computed as $\gamma^{v} c_{1} g_{c}^{\sigma} \cdot c_{2} \cdot g_{c}^{e_{c}}$ which is equal to $\gamma^{v} c g_{c}^{f\left(h_{c}^{c}\right)}$ for $c=c_{1} c_{2} \cdot \bar{A}$ and $\bar{B}$ are computed in a similar way, and the factor $f_{2}\left(e_{c}, e_{b}\right)$ is put into $\bar{A}$. The following relations hold between the blinded numbers and their unblinded values:

$$
\begin{aligned}
& \bar{C}=\gamma^{v} C \\
& \bar{A}=\alpha^{v} A^{1 / k_{1}} \\
& \bar{B}=\beta^{v} B
\end{aligned}
$$

The bank then chooses a random $k_{2}$, and sends $c_{2}, b_{2}, k_{2},\left(\bar{C}^{k_{2}} \cdot \bar{A}\right)^{1 / v}$, and $\left(\bar{C}^{U} \cdot \bar{B}\right)^{1 / v}$ to Alice.

5. Using $c_{2}$ and $b_{2}$ Alice can compute $c$ and $b$ as $c_{1} c_{2}$ and $b_{1} b_{2}$ respectively. She now constructs the numbers $C, A$, and $B$ as $c g_{c}^{f\left(h_{c}^{c}\right)}, a g_{a}^{f(a)}$, and $b g_{b}^{f\left(h_{b}^{b}\right)}$. Alice computes the first signature $S_{a}$ as $\left(\left(\bar{C}^{k_{2}} \cdot \bar{A}\right)^{1 / v} / \gamma^{k_{2}} \alpha\right)^{k_{1}}$, and the second signature $S_{b}$ as $\left(\bar{C}^{U} \cdot \bar{B}\right)^{1 / v} / \gamma^{U} \beta$. The $k_{1}$ 'th power is needed because 
the base number $a$ was chosen as $\left(a_{1} a_{2}\right)^{k_{1}}$ instead of $a_{1} a_{2}$. All the necessary adjustments in the exponent of $g_{a}$ were already made by Alice. The total effect of this $k_{1}$ 'th power is to get a $v^{\prime}$ th root on the number $C^{k} A$ where $k=k_{1} k_{2}$.

Finally Alice checks that the signatures she received are correct by verifying that $S_{a}^{v}=C^{k} A$ and $S_{b}^{v}=C^{U} B$.

Alice ends up with the following set of numbers: $c, a, b, k, S_{a}$, and $S_{b}$ which are the 3 base numbers, the random parameter for the secret sharing line and the 2 signatures. These 6 numbers plus the identity $U$ are used as input to the payment protocol.

We still need a few additions to this protocol to protect Alice against framing by the Bank. To this end we let $U$ be the concatenation of Alice's identity and a unique coin number. This makes the $U$ 's of all the coins distinct. Secondly, in the third transmission Alice includes a digital signature on $U$ and all the data in the first three transmissions. If the bank now claims that Alice spent a coin twice, it must show a transcript of the withdrawal protocol for that coin. This transcript must include the correct data in the last transmission. (This is verifiable by a third party.) The bank also shows $a, b$ and $c$ from the doubly spent coin.

If Alice didn't spend the coin with that identity twice, then the bank can have no knowledge regarding $a, b$, or $c$. So if the bank tries to frame Alice, the triple $(a, b, c)$ will (with high probability) be different from the actual values used by Alice. If Alice can provide a different triple $(a, b, c)$ plus the corresponding blinding factors that match the transcript, then the bank must be framing Alice, as she cannot generate a new triple which matches a given transcript.

\section{Remarks}

It would be nice to make a similar system where $C, A$, and $B$ are images under oneway functions of a single base number $c$. If $c$ can also be made smaller (say in $\mathbb{Z}_{v}$ instead of $\mathbb{Z}_{n}$ ) then we could store a coin in about 70 bytes. However, constructing an efficient withdrawal protocol for such a coin remains an open problem.

Work is currently under way to implement this scheme on workstations to provide e-mail money. An extension of this coin system to $n$-spendable coins (which can be spent $n$ times but not $n+1$ times) and the incorporation of observers in the coin system are described in [Fer94].

\section{References}

[Bra93] Stefan Brands. An efficient off-line electronic cash system based on the representation problem. Technical Report CS-R9323, CWI (Centre for Mathematics and Computer Science), Amsterdam, 1993. Anonymous ftp: ftp.cwi.nl:/pub/CWIreports/AA/CS-R9323.ps.Z. 
[Bra94] Stefan Brands. Electronic cash systems based on the representation problem in groups of prime order. In Proceedings of CRYPTO '93, 1994. To appear.

$\left[\mathrm{CdBvH}^{+}{ }^{90}\right]$ David Chaum, Bert den Boer, Eugène van Heyst, Stig Mjølsnes, and Adri Steenbeek. Efficient off-line electronic checks. In J.-J. Quisquater and J. Vandewalle, editors, Advances in Cryptology-EUROCRYPT' '89, Lecture Notes in Computer Science, pages 294-301. Springer-Verlag, 1990.

[CFN90] David Chaum, Amos Fiat, and Moni Naor. Untraceable electronic cash. In S. Goldwasser, editor, Advances in Cryptology-CRYPTO '88, Lecture Notes in Computer Science, pages 319-327. Springer-Verlag, 1990.

[Cha90] David Chaum. Online cash checks. In J.-J. Quisquater and J. Vandewalle, editors, Advances in Cryptology-EUROCRYPT' 89 , Lecture Notes in Computer Science, pages 288-293. Springer-Verlag, 1990.

[Cha92] David Chaum. Randomized blind signature. Personal communications, April 1992.

[EvH92] Jan-Hendrik Evertse and Eugène van Heyst. Which new RSA-signatures can be computed from certain given RSA-signatures? J. Cryptology, $5(1): 41-52,1992$.

[Fer93] Niels Ferguson. Single term off-line coins. Technical Report CS-R9318, CWI (Centre for Mathematics and Computer Science), Amsterdam, 1993. Anonymous ftp: ftp.cwi.nl:/pub/CWIreports/AA/CS-R9318.ps.Z.

[Fer94] Niels Ferguson. Extensions to single term off-line coins. In Proceedings of CRYPTO '93, 1994. To appear.

[FY93] Matthew Franklin and Moty Yung. Secure and efficient off-line digital money. In A. Lingas, R. Karlsson, and S. Carlsson, editors, Automata, Languages and Programming, 20th International Colloquium, ICALP 93, Lund, Sweden, Lecture Notes in Computer Science 700, pages 265-276. Springer-Verlag, 1993.

[Hir93] Rafael Hirschfeld. Making electronic refunds safer. In Advances in Cryptology-CRYPTO '92, 1993. To appear.

[OO92] Tatsuaki Okamoto and Kazuo Ohta. Universal electronic cash. In J. Feigenbaum, editor, Advances in Cryptology-CRYPTO '91, Lecture Notes in Computer Science, pages 324-337. Springer-Verlag, 1992.

[RSA78] Ronald Rivest, Adi Shamir, and Leonard Adleman. A method for obtaining digital signatures and public-key cryptosystems. Communications of the $A C M, 21: 120-126$, February 1978.

[Sha79] Adi Shamir. How to share a secret. Communications of the ACM, 22(11):612-613, 1979.

[vA90] Hans van Antwerpen. Off-line electronic cash. Master's thesis, Eindhoven University of Technology, department of Mathematics and Computer Science, 1990. 